\title{
Stage III Lung Cancer AJCC v6
}

National Cancer Institute

\section{Source}

National Cancer Institute. Stage III Lung Cancer A/CC v6. NCI Thesaurus. Code C8773.

Stage III includes: IIIA (T1, N2, M0); (T2, N2, M0); (T3, N1,M0); (T3, N2, M0) or IIIB (Any T, N3, M0); (T4, Any N, M0). N1: Metastasis to ipsilateral peribronchial and/or ipsilateral hilar lymph nodes, and intrapulmonary nodes including involvement by direct extension of the primary tumor. N2: Metastasis to ipsilateral mediastinal and/or subcarinal lymph node(s). N3: Metastasis to contralateral mediastinal, contralateral hilar, ipsilateral or contralateral scalene, or supraclavicular lymph node(s). T1: Tumor is $3 \mathrm{~cm}$ or less in greatest dimension, surrounded by lung or visceral pleura, and without bronchoscopic evidence of invasion more proximal than the lobar bronchus. T2: Tumor has any of the following features of size or extent: more than $3 \mathrm{~cm}$ in greatest dimension; involves the main bronchus, $2 \mathrm{~cm}$ or more distal to the carina; invades the visceral pleura; associated with atelectasis or obstructive pneumonitis that extends to the hilar region but does not involve the entire lung. T3: Tumor (of any size) directly invades any of the following: chest wall (including superior sulcus tumors), diaphragm, mediastinal pleura, parietal pericardium; or tumor in the main bronchus less than $2 \mathrm{~cm}$ distal to the carina but without involvement of the carina; or associated atelectasis or obstructive pneumonitis of the entire lung. T4: Tumor of any size that invades any of the following: mediastinum, heart, great vessels, trachea, esophagus, vertebral body, carina; or separate tumor nodules in the same lobe; or tumor with a malignant pleural effusion. M0: No distant metastasis. (AJCC 6th ed.) - 2003 